\title{
Automatic Classification System of Electric Parts Focused on Periodicity of Leads
}

\author{
Shuichiro Kito ${ }^{\mathrm{a},{ }^{*}}$, Koosuke Hattori ${ }^{\mathrm{b}}$, Ryo Taguchi ${ }^{\mathrm{a}}$, Taizo Umezaki ${ }^{\mathrm{a}}$ \\ a Nagoya Institute of Technology, Gokiso-cho, Nagoya, Aichi 466-8555, Japan \\ ${ }^{\mathrm{b}}$ Chubu University, 1200 Matsumoto-cho, Kasugai, Aichi 487-8501, Japan \\ *Corresponding Author: kitou@ume.mta.nitech.ac.jp
}

\begin{abstract}
Positioning and inspection methods by image processing are necessary for surface mount technology to mount various surface mount devices (parts) onto PCBs. The positioning and inspection methods are prepared for each category of parts. The category of the parts is usually chosen by manual operation based on the experience and intuition of a worker, this means that ambiguity will be included. Our research focused on connectors that had low automatic classification rate in previous research. We are proposing a method by using SVM with HLAC features on power spectrum images to detect the region of lead groups in part images. The experimental results show that the proposed method has improved classification performance of the connectors (115 samples) from 40.0[\%] to $91.3[\%]$. For all kinds of parts (748 samples), classification performance has improved from $89.2[\%]$ to $96.0[\%]$.
\end{abstract}

Keywords: Automatic classification, SMD, SVM, HLAC.

\section{Introduction}

Surface mounted devices (SMDs) used in surface mount technology are created in various forms according to the miniaturization and diversification of electronic devices. SMDs (hereafter called parts) are imaged by a camera during the placement process onto a printed circuit board (PCB), and positioning processes and inspection processes are performed. Some image processing algorithms are prepared based on the information of the form of the parts, the arrangement pattern of electrodes, etc., and it is necessary to choose suitable algorithms for the positioning methods and inspection methods according to the kind of parts. For example, it is important to select the algorithm not only to detect the position, but also to check for the presence of leads and measure bending of each lead for IC parts shown in Fig. 1(a). For chip parts, since a large amount of chip parts are mounted onto a PCB, it is important to select an algorithm with high speed and high precision.

In recent years, since labor saving has been required from the point of view of reducing board production cost, the automatic classification method of SMD types is required to reduce the number of steps by hand as much as possible. It is usual to select a suitable algorithm for the SMD based on the design diagram. However, there are cases of reselecting the suitable algorithm such as due to luminance information for parts that is unable to be obtained from design diagrams. In this case, the algorithm is selected based on the experience and intuition of a specialized person.

In the previous research, the classification method of the hierarchical type which used DP matching was proposed ${ }^{(1}$, 2). Although high classification performance is shown for general parts such as ICs, chips, minimolds, connectors, and BGAs as shown in Fig. 1(a) to (e) by this method, complex connector parts as shown in Fig, 1 (g), (h) are classified as odd form parts such as Fig.1 (f). Especially in these parts, despite to have many leads which are typical form for SMDs, there is a problem that the algorithm for detecting bending or presence of leads is not selected. To improve classification performance, we are proposing a method using Support Vector Machine (SVM) based on Higher-order Local AutoCorrelation (HLAC) features from power spectrum images to classify SMDs that are not classified correctly by previous research.

\section{System Overview}

The configuration of imaging system is shown in Fig. 2. 


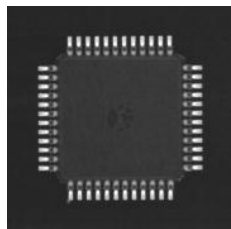

(a) IC
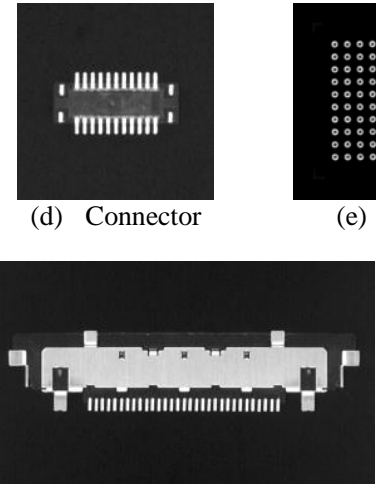

(g) Complex part (connector) (d) Connector

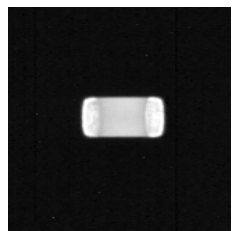

(b) Chip

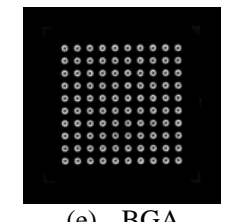

(e) BGA

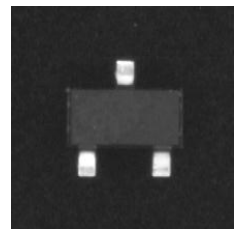

(c) Minimold

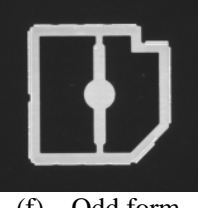

(f) Odd form
Fig. 1. Example of part images.

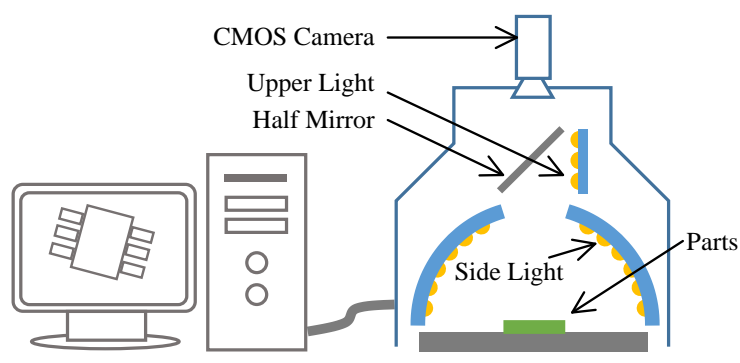

Fig. 2. System components.

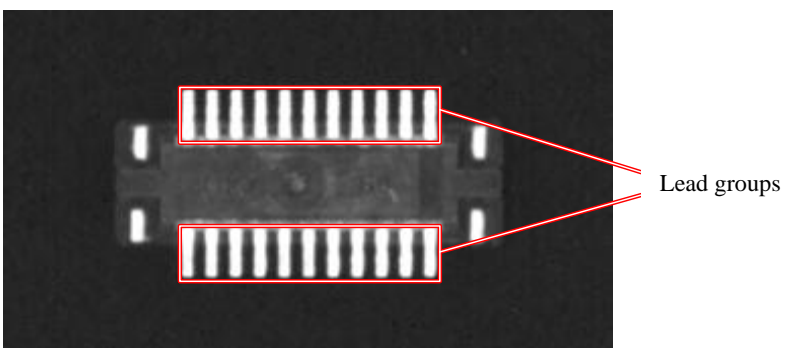

Fig. 3. Lead groups examples.

Table 1. Categorizing results (previous research).

\begin{tabular}{|c|c|c|c|c|c|c|c|c|c|}
\hline & \multirow{2}{*}{ Part Categories } & \multicolumn{6}{|c|}{ Result } & \multirow{2}{*}{ Total } & \multirow{2}{*}{ Accuracy $[\%$} \\
\hline & & Chip & Minimold & IC & BGA & Connector & Odd form & & \\
\hline \multirow{6}{*}{$\begin{array}{l}\text { 音 } \\
\text { : }\end{array}$} & Chip & 162 & & & & & 1 & 163 & 99.4 \\
\hline & Minimold & 4 & 139 & & & & 3 & 146 & 95.2 \\
\hline & IC & & & 100 & & & 1 & 101 & 99.0 \\
\hline & BGA & 2 & & & 104 & & & 106 & 98.1 \\
\hline & Connector & & & 4 & & 46 & 65 & 115 & 40.0 \\
\hline & Odd form & & 1 & & & & 116 & 117 & 99.1 \\
\hline \multicolumn{2}{|r|}{ Total } & & & & & & & 748 & 89.2 \\
\hline
\end{tabular}

This configuration is similar to that of previous research ${ }^{(1,2)}$, and the imaging device is attached to a computer. This camera can image with a resolution of $2000 \times 2000$ [pixels] with 8bit gray scale. There are two types of light sources in order to equalize the imaging environment, one is the incident light source for projecting light on the part upper surface from the optical axis of the camera by a half mirror. The other is the side light source for projecting light from an oblique direction of the part. Usually, the incident light source is used, and if there are protruding electrodes such as on a BGA, the side light source is used. Fig. 1(e) shows an example image of a BGA using a side light source.

\section{Experimental Data}

At first, we perform an evaluation by creating an automatic classifier for SMD types BGAs, ICs, minimolds, connectors, chips and odd forms using the method based on the previous research ${ }^{(1,2)} .748$ sample images were used for the experiment which were categorized by a person in advance. We measured the accuracy rate of the categories that the automatic classifier determined. The experimental results are shown in Table 1. There are recognition rates over 95[\%] for parts other than connectors, and rates as low as $40[\%]$ for connectors. Connectors are parts used to connect electrodes and they are created in a wide variety of shapes according to the size and design of the final product. Therefore, if the pixel frequency and the zero-crossing frequency of the whole part region proposed in the previous research ${ }^{(1,2)}$ are used as features, the DP distance from the reference is increased by luminance changes such as the internal structure of the part. It cannot be classified correctly due to the increased distance to the reference in the subspace method.

\section{Proposed Method}

In this paper, we focus on periodicity of leads location to distinguish connectors and odd form parts. To obtain periodic features, we create power spectrum images from 


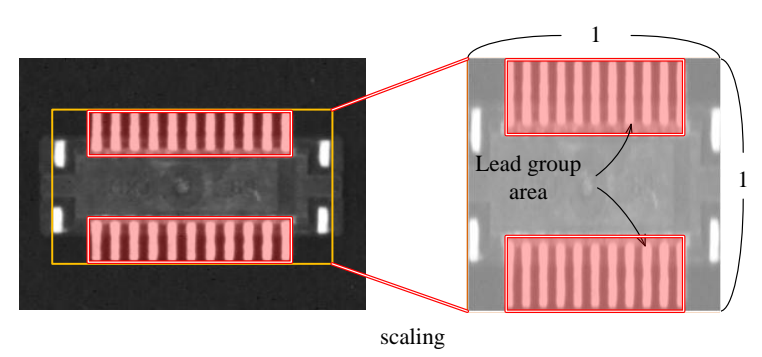

(a) Scaling image.

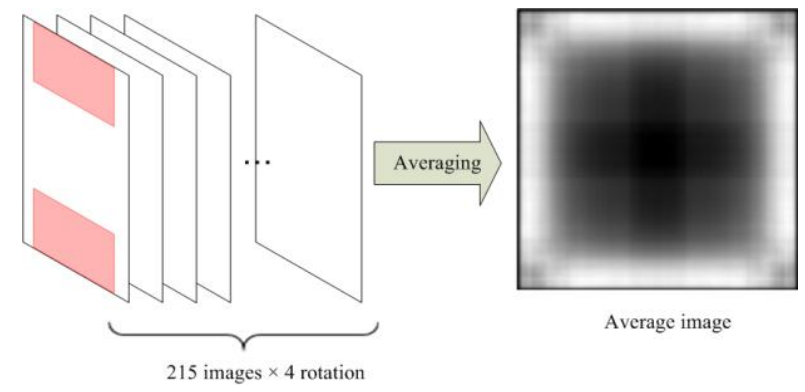

(b) Averaging scaling images.

Fig. 4. Procedure to get the average image.

part images, and distinguish the existence of leads by a classifier using SVM with HLAC features obtained from these images. The regions for applying FFT were defined by using the existence probability of leads.

\subsection{The Existence Region of Lead Groups}

Leads are one of the typical electrode shapes for SMDs, and they are usually attached pointing away from the body

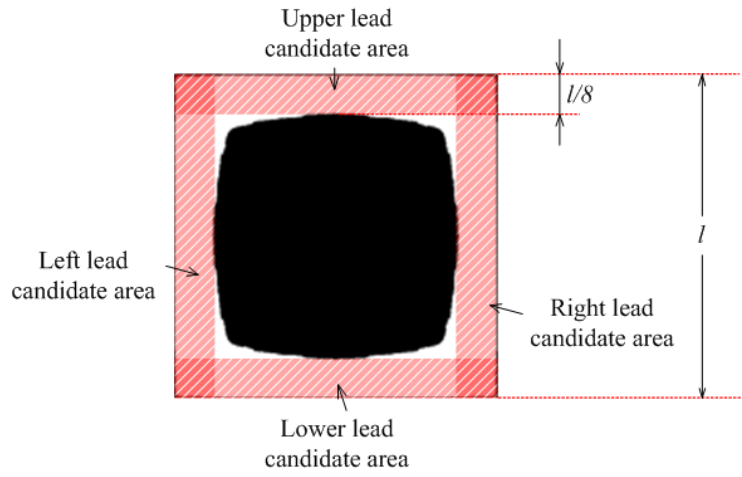

Fig. 5. Identification of lead candidate region.

of the part. Therefore, it is expected that the existence probability of leads is high in the vicinity of the outer periphery of parts. On the other hands, these probabilities in the center of parts are low. In many parts, multiple leads are usually attached side by side in the same direction. In this research, these lined up leads are treated as a group and named "Lead Group" (Fig. 3). We measured sizes and positions of lead groups in the part images whose lead groups were defined in advance. Because the orientation of the supplying direction $(0,90,180,270$ degrees) is determined by the user, the correct orientation of the part is not able to be determined by an image. Hence we rotate part images in four directions, and generate an image of the lead group region by voting the region of the defined lead group. We normalized the part region to be a square area $(512 \times 512$ pixels $)$, and

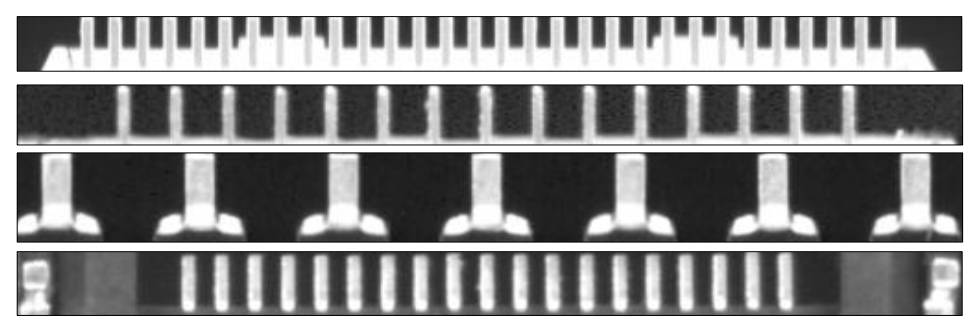

(a) Images of lead candidate area.

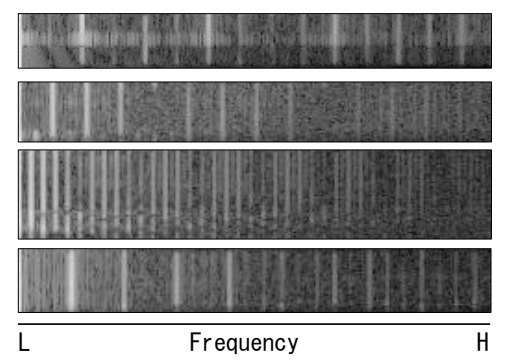

(b) Power spectrum images.

Fig. 6. Example of positive (lead group) images.

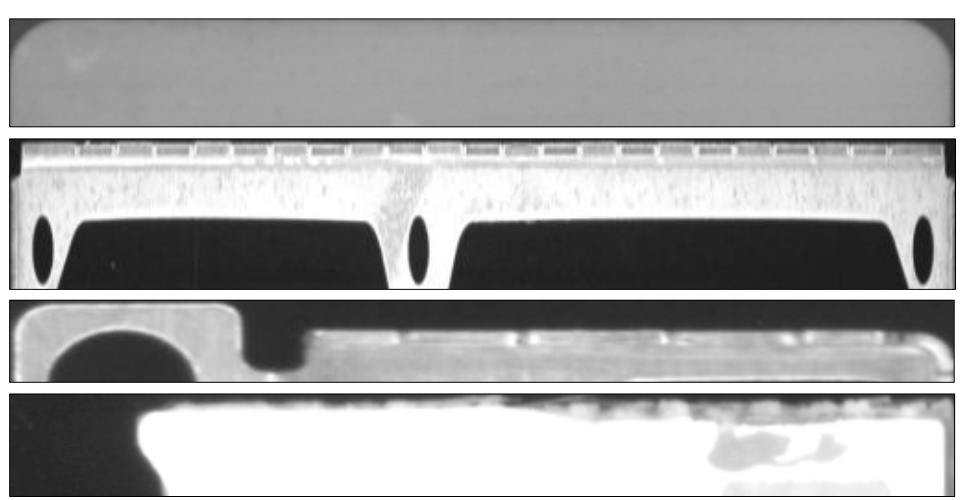

(a) Images of lead candidate area.

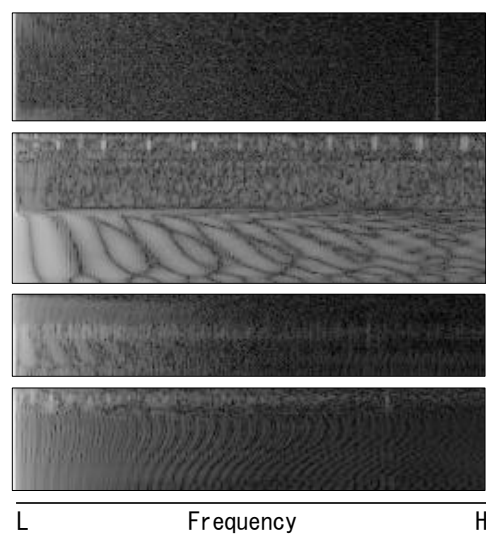

(b) Power spectrum images.

Fig. 7. Example of negative (non lead group) images. 


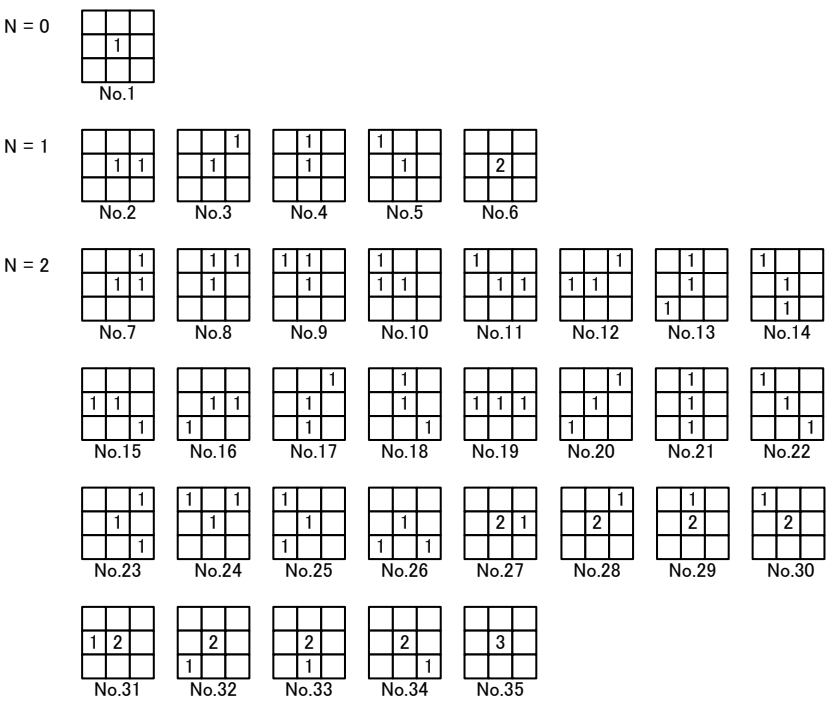

Fig. 8. Mask pattern of HLAC.

imaged by normalizing the maximum number of votes of a lead group area to be luminance 255. 215 samples of part images with the lead groups were used for evaluation. The procedure for creating a lead group area image and an averaged image obtained is shown in Fig. 4. It is confirmed that the lead groups are concentrated in the peripheral portion of the part region. An image that is binarized by a discriminant analysis method is shown in Fig. 5. From the binarized image, we defined the image cut out one eighth length of the region as the lead candidate images. These images are used in order to discriminate the existence of lead groups.

\subsection{Periodic Feature Extraction}

Based on the results of 4.1, the examples of lead candidate images acquired from part images which were classified as odd form in previous researches are shown in Fig. 6 (a), Fig. 7 (a). These images are displayed by applying rotation correction so that the outside should be upward. The images shown in Fig. 6 (a) are examples of regions including leads, the images shown in Fig. 7 (a) are examples of regions without leads. Periodic brightness changes are found in the horizontal direction in Fig. 6 (a). A power spectrum image is obtained by applying FFT to the lead candidate images for each horizontal line. Since the number of data needed to apply FFT is a power of 2, the lead candidate image shown in Fig. 6 (a), 7 (a) are extended to have 512 [pixels] in the width direction. The obtained power spectrum images are shown in Fig. 6 (b) and Fig. 7 (b). Periodic vertical lines are shown in the power spectrum images with lead groups (Fig. 6(b)), whereas they are not shown in the images without lead groups (Fig. 7(b)).
In this paper, we are proposing a method of determining whether the part has a lead group or not by using HLAC features obtained from the power spectrum image. HLAC is used in various image processing such as character recognition ${ }^{(4)}$ or face recognition ${ }^{(5,6)}$, and it is proposed as a feature that is highly versatile. The $N$-th order autocorrelation function with $N$ displacements $a_{1}, \cdots, a_{N}$ are defined as the following function.

$$
x\left(a_{1,} \cdots, a_{N}\right)=\int I(r) I\left(r+a_{1}\right) \cdots I\left(r+a_{N}\right) d r
$$

where $r$ is a referred pixel coordinate, $I(r)$ is the intensity value at $r$. The number of combinations of the displacement vectors is the dimension of the feature vector. Then, when the order $N$ is restricted up to the second and the range of displacements is restricted within a local $3 \times 3$ window, the numbers of patterns of the displacement are reduced to 35 in the gray scale image. Fig. 8 shows the mask patterns of HLAC features.

It is well known that HLAC has additive and shiftinvariant features. As to the vertical stripes of the power spectral image shown in Fig. 6(b), occurrence position is determined by the pitch between leads. It is considered that the use of property of the shift-invariant feature of HLAC is suitable for recognition, because it is possible to obtain the features of the independent pitch between the lead and extension of the data when applying FFT. We used features by normalizing the area of the spectrum image obtained from HLAC features, since the height of the power spectrum image varies depending on the area in the part image.

\subsection{Classification Method with SVM}

In order to determine whether a lead group is contained in the lead candidate image, we use Support Vector Machine (SVM) with a reputation for binary classification of highdimensional data ${ }^{(7,8)}$ by using the method shown in 4.2. In consideration of overlapping regions between classes, we use the soft margin SVM with RBF kernel. Given $\left(x_{n}, t_{n}\right)$ as a set of labels and training data, $\xi_{n}$ as slack variables, $C$ as penalty, optimization issue of SVM is expressed as follows.

$$
\begin{aligned}
& \min _{\mathbf{w}, b, \xi} C \sum_{n} \xi_{n}+\frac{1}{2}\|\mathrm{w}\|^{2} \\
& \text { subject to } t_{n}\left(\mathrm{w}^{\mathrm{T}} \phi\left(x_{n}\right)+b\right) \geq 1-\xi_{n} \\
& \xi_{n} \geq 0
\end{aligned}
$$

RBF kernel is expressed as

$$
K\left(\mathrm{x}, \mathrm{x}^{\prime}\right)=\exp \left(-\gamma\left\|\mathrm{x}-\mathrm{x}^{\prime}\right\|^{2}\right)
$$

where $\gamma$ is a parameter. Since the adjustment of 
Table 2. Categorizing results (proposed).

\begin{tabular}{|c|c|c|c|c|c|c|c|c|c|}
\hline & \multirow{2}{*}{ Part Categories } & \multicolumn{6}{|c|}{ Result } & \multirow{2}{*}{ Total } & \multirow{2}{*}{ Accuracy $[\%]$} \\
\hline & & Chip & Minimold & IC & BGA & Connector & Odd form & & \\
\hline \multirow{6}{*}{ 言 } & Chip & 162 & & & & & 1 & 163 & 99.4 \\
\hline & Minimold & 4 & 139 & & & & 3 & 146 & 95.2 \\
\hline & IC & & & 100 & & & 1 & 101 & 99.0 \\
\hline & BGA & 2 & & & 104 & & & 106 & 98.1 \\
\hline & Connector & & & 4 & & 105 & 6 & 115 & 91.3 \\
\hline & Odd form & & 1 & & & 8 & 108 & 117 & 92.3 \\
\hline & Total & & & & & & & 748 & 96.0 \\
\hline
\end{tabular}

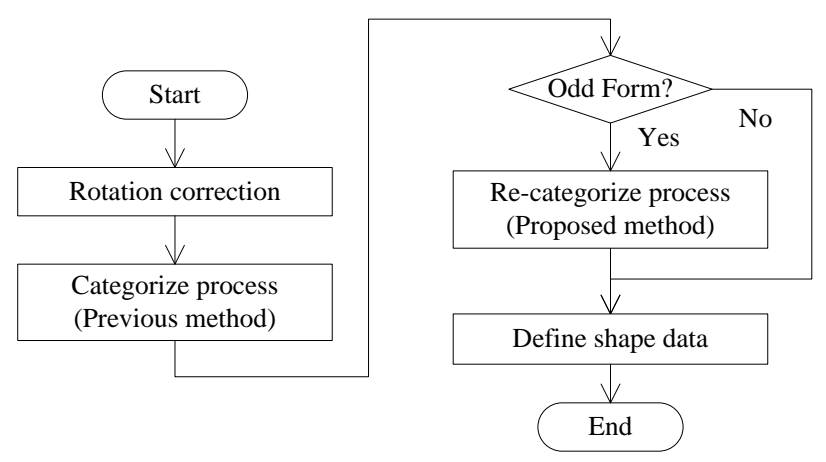

Fig. 9. Outline of processing flow.

parameters $C, \gamma$ affects the discrimination ability, we perform the $\mathrm{n}$-fold cross validation using the training data, to determine the optimum $C, \gamma$.

\subsection{Integration Into the Automatic Classifier}

Hierarchical classifier was proposed in previous research ${ }^{(1,2)}$. In this classifier, there are many misclassifications of connector images, and in these cases, they are classified as odd form. So, for part images classified as odd form, we improve classification performance by adding a process to classify as connectors when a lead group for any of the lead candidate images were present in the area of the top, bottom, left, or right. The outline of the processing flow is shown in Fig. 9.

\section{Evaluation Experiment}

\subsection{Discrimination Performance of Lead Candidate Images}

Based on the lead candidate region determined by the method in 4.1, we get the lead candidate images from parts with leads and odd form parts. Then, we classify them into positive samples (with leads) of 441 images, and negative samples (without leads) of 737 images on visual observation. We obtain the accuracy rate by performing a 10 -fold cross validation on these images. In order to confirm effectiveness of the features obtained in the frequency domain, we

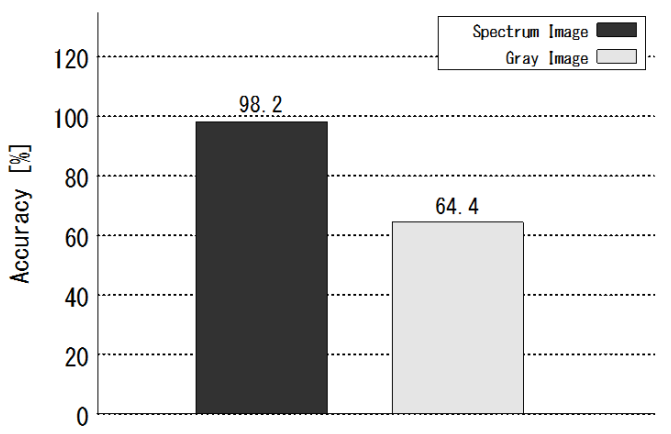

Fig. 10. Accuracy for lead image discrimination.

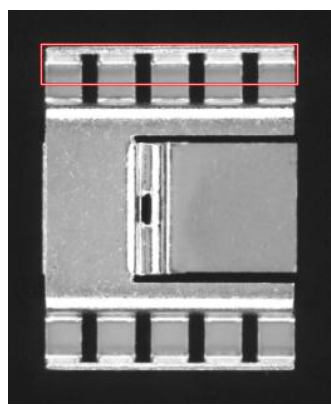

(a) The entire part of connector. (In Red : lead candidate region)

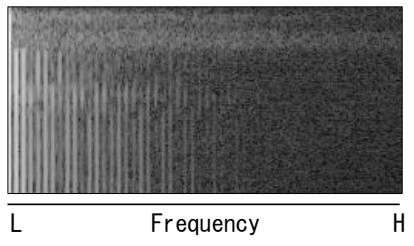

(b) Power spectrum image of red region.
Fig. 11. Sample of false positive image.

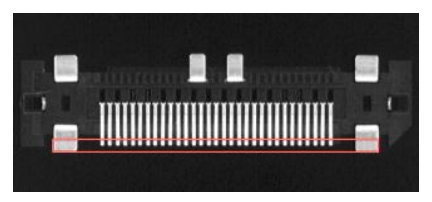

(a) The entire part of connector. (In Red : lead candidate region)

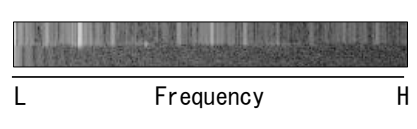

(b) Power spectrum image of red region.
Fig. 12. Sample of false negative image.

compare with the case of obtained HLAC feature on the original image. The experimental results are shown in Fig. 10. This results show that it is effective to obtain the feature in frequency domain.

Although shift-invariant features of lead shapes can be obtained by HLAC features in the original image, it is not possible to obtain periodic features by arrangement of leads. Since periodic features are obtained by FFT+HLAC, 
Table 3. Processing time.

\begin{tabular}{c|c|c}
\hline & $\begin{array}{c}\text { Categorize process } \\
\text { (748 samples) }\end{array}$ & $\begin{array}{c}\text { Re-categorize process } \\
\text { (186 samples) }\end{array}$ \\
\hline Average [ms] & 289.0 & 49.1 \\
\hline Max [ms] & 2509.3 & 236.6 \\
\hline Min [ms] & 11.0 & 2.5 \\
\hline
\end{tabular}

proposed method is more effective than simple HLAC features.

\subsection{Performance Evaluation as an Automatic Classifier}

Table 2 shows the classification results for the proposed method. Discrimination performance has improved from $40.0[\%]$ to $91.3[\%]$ for connector parts. Although misclassification of IC parts still remains, this misclassification is not problematic because both the positioning and inspection processes for connector and IC parts use lead information.

On the other hand, misclassifying actual odd form parts as connectors has increased. An example of a misclassification of an odd form part as a connector is shown in Fig. 11. It is because the object has periodically line up shapes located in the outer periphery of the part region, so it was misclassified. It can be recognized though as an odd form in a post-process with a lead identification process.

Misclassifying connectors as odd form still remained as 8.7[\%]. An example of a misclassification image is shown in Fig. 12. As can be seen from Fig. 12, lead groups account for only about half the region of the image, and it is assumed that the features of periodic vertical stripes decreased. For this misclassification, there is room to make improvement, such as a review of the acquisition method for the lead candidate region.

Computational times of our proposed method are shown in Table 3 with Corei7 @2.1GHz. The re-categorize process (proposed method) in Fig. 9 re-classifies odd forms categorized by the categorize process (previous method) into connectors and odd forms. In this experiment, an average of computational times on the categorize process was 289.0 [ms]. An average of computational times was taken 49.1 $[\mathrm{ms}]$ in the re-categorized process. Since this processing time is sufficiently shorter than the previous method, proposed method is usable as an additional process.

\section{Conclusions}

We have shown that the classification performance of the connector parts is improved from 40.0[\%] to $91.3[\%]$ by using the proposed method for SMDs that were classified as odd form in the previous research. Classification performance of the overall parts has improved from 89.2[\%] to 96.0 [\%]. By using SVM with HLAC feature for the power spectrum image in the lead candidate region, we have shown high performance in the SMD image. On the other hand, for connectors in which a lead group only exists in the center of part region, there were cases of misclassifying these as odd form. To build a more accurate classifier by reviewing the lead candidate region and using a method such as combining the identification of the shape of the lead is something to do in the future.

\section{References}

(1) H.Esaki, K.Kagii, T.Umezaki, T.Horikosi: "Hierarchical Automatic Classification System of Electronic Parts", The transactions of the Institute of Electrical Engineers of Japan. C, A publication of Electronics, Information and System Society 126(12), 1447-1453, 2006-12-01

(2) H.Esaki , " Research on the Application of Image Recognition Technology in the Electronic Devices Chapter 2. Classification System of Electronic Devices" (In Japanese), doctor thesis, 2007

(3) H.Esaki, K.Kagii, T.Umezaki, T.Horikosi: "Automatic Generation of IC Part Shape Data" , The transactions of the Institute of Electrical Engineers of Japan. D, A publication of Industry Applications Society 127(2), 152-157, 2007-02-01

(4) N.Otu, "Mathematical Studies on Feature Extraction in Pattern Recognition" (In Japanese) Research of the ETL, No 818, 1981

(5) F. Goudail, E. Lange, T. Iwamoto, K. Kyuma, N. Otsu, “ Face Recognition System Using Local Autocorrelations and Multiscale Integration", IEEE Transactions on Pattern Analysis and Machine Intelligence, VOL. 18, NO. 10, OCTOBER 1996.

(6) T. Kurita, N. Otsu, T. Sato, "A Face Recognition Method Using Higher Order Local Autocorrelation And Multivariate Analysis" , Proc. International Conference on Pattern Recognition, Vol.2, p.213-216, 1992

(7) N. Dalal, B. Triggs, "Histograms of oriented gradients for human detection", CVPR05, 2005.

(8) F. Han, Y. Shan, R. Cekander, "A Two-Stage Approach to People and Vehicle Detection with HOG-Based SVM", PerMIS, pp. 133-140, 2006. 